$\begin{array}{cl}\begin{array}{cl}\text { Revue } \\ \text { de Ihistoire }\end{array} & \text { Revue de l'histoire des religions } \\ \text { des religions } & \begin{array}{l}4 \mid 2012 \\ \text { Varia }\end{array}\end{array}$

\title{
Albertus BERNABÉ (éd.), Poetae Epici Graeci. Pars II. Orphicorum et Orphicis similium testimonia et
} fragmenta

- Fasc. 1. Bibliotheca Teubneriana, Monachii et Lipsiae : In Aedibus K.

G. Saur, 2004, LXXXV + 394 p. ISBN 3-598-71707-5 79,95€ (\$112). - Fasc. 2. Bibliotheca Teubneriana, Monachii et Lipsiae : In Aedibus K. G. Saur, 2005, XXV + 553 p. ISBN 3-598-71708-3 79,95€ (\$112.00). - Fasc. 3.

Musaeus. Linus. Epimenides. Papyrus Derveni. Indices. Bibliotheca Teubneriana, Berolini et Novi Eboraci : Walter de Gruyter, 2007, XXV + 465 p. ISBN 978-3-11-019487-6 79,95€ (\$112)

Jean-Michel Roessli

\section{(2) OpenEdition}

\section{Édition électronique}

URL : http://journals.openedition.org/rhr/7992

DOI : $10.4000 /$ rhr.7992

ISSN : 2105-2573

Éditeur

Armand Colin

Édition imprimée

Date de publication : 1 décembre 2012

Pagination : 534-537

ISBN : 978-2200-92796-7

ISSN : 0035-1423

Référence électronique

Jean-Michel Roessli, «Albertus bernabé (éd.), Poetae Epici Graeci. Pars II. Orphicorum et Orphicis similium testimonia et fragmenta », Revue de l'histoire des religions [En ligne], 4 | 2012, mis en ligne le 24 janvier 2013, consulté le 22 septembre 2020. URL : http://journals.openedition.org/rhr/7992 ; DOI : https:// doi.org/10.4000/rhr.7992

Ce document a été généré automatiquement le 22 septembre 2020.

Tous droits réservés 


\section{Albertus BERnABÉ (éd.), Poetae Epici Graeci. Pars II. Orphicorum et Orphicis similium testimonia et fragmenta}

- Fasc. 1. Bibliotheca Teubneriana, Monachii et Lipsiae : In Aedibus K. G. Saur, 2004, LXXXV + 394 p. ISBN 3-598-71707-5 79,95€ (\$112). - Fasc. 2. Bibliotheca Teubneriana, Monachii et Lipsiae : In Aedibus K. G. Saur, 2005, XXV + 553 p. ISBN 3-598-71708-3 79,95€ (\$112.00). - Fasc. 3. Musaeus. Linus. Epimenides. Papyrus Derveni. Indices. Bibliotheca Teubneriana, Berolini et Novi Eboraci : Walter de Gruyter, 2007, XXV + 465 p. ISBN 978-3-11-019487-6 79,95€ (\$112)

Jean-Michel Roessli

\section{RÉFÉRENCE}

Albertus Bernabé (éd.), Poetae Epici Graeci. Pars II. Orphicorum et Orphicis similium testimonia et fragmenta - Fasc. 1. Bibliotheca Teubneriana, Monachii et Lipsiae : In Aedibus K. G. Saur, 2004, LXXXV + 394 p. ISBN 3-598-71707-5 79,95€ (\$112). - Fasc. 2. Bibliotheca Teubneriana, Monachii et Lipsiae : In Aedibus K. G. Saur, 2005, XXV + 553 p. ISBN 3-598-71708-3 79,95 € (\$112.00). - Fasc. 3. Musaeus. Linus. Epimenides. Papyrus Derveni. Indices. Bibliotheca Teubneriana, Berolini et Novi Eboraci : Walter de Gruyter, 2007, XXV + 465 p. ISBN 978-3-11-019487-6 79,95€ (\$112)

Cette nouvelle édition des fragments et témoignages orphiques était attendue depuis longtemps et nul doute qu'elle remplacera durablement l'édition d'Otto Kern, qui s'était imposée comme édition de référence depuis 1922 (Orphicorum fragmenta, Berlin : Weidmann, [réimpr. Zurich 1970]), mais qui s'est rapidement révélée insuffisante, principalement en raison des nouvelles découvertes et de l'explosion des publications relatives à ce sujet. Cette nouvelle édition monumentale en trois volumes a pour ambition de réunir tous les fragments et témoignages relatifs à Orphée et aux pratiques 
religieuses et littéraires associées à son nom. Si le choix, l'organisation et la contextualisation des fragments orphiques adoptés par Alberto Bernabé peuvent faire débat, la mise à jour du matériel orphique et la fantastique couverture bibliographique qu'elle offre font de cette édition le point de départ de toute recherche future sur Orphée et l'orphisme et cela, malgré les nombreuses petites imperfections et coquilles qu'elle contient.

2 À la différence de Kern, qui faisait commencer son édition avec les témoignages relatifs à Orphée et repoussait dans la deuxième partie de l'ouvrage les fragments orphiques, c'est-à-dire les textes décrivant les rites, pratiques et croyances orphiques, de même que les écrits attribués à Orphée et ses épigones, Bernabé inverse les choses et inaugure son édition avec les fragments orphiques (F pour fragmenta), qui occupent le premier volume et une grosse partie du deuxième, alors que les témoignages ( $\mathrm{T}$ pour testimonia) sont relégués dans la dernière partie de l'édition, à laquelle Bernabé prend soin d'ajouter une troisième catégorie, absente de Kern, les vestigia (V), où sont rangés tous les textes qui portent des traces d'influence orphique, sans être des textes orphiques à proprement parler. Ce renversement dans l'organisation de la matière a pour conséquence de mettre l'accent sur le matériel orphique et de reléguer tout ce qui a trait à la figure d'Orphée au second plan. Cette option, qui peut tout à fait se défendre, reflète l'orientation prise par la recherche au cours des deux ou trois dernières décennies, où l'intérêt pour les idées et pratiques religieuses associées au chantre thrace l'ont emporté sur la figure mythologique qui en est l'inspiratrice.

3 Le premier volume des Orphica réunit tout ce qui a trait aux théogonies orphiques (frg. 1-378), aux mythes de Déméter et Perséphone (frg. 379-402), à la nature du monde (frg. 403-420) et au destin de l'âme (frg. 421-469). Bernabé fait débuter son édition avec les théogonies orphiques (frgs. 1-378), en suivant une chronologie descendante et en commençant donc par celle de Derveni, d'Eudème, d'Hellanicos et Hiéronymos, pour finir avec la théogonie des Rhapsodies, quelques matériaux difficiles à classer et l'imitation juive d'un discours sacré, appelée Diathekai, Testament d'Orphée par les auteurs paléochrétiens (sur ce dernier texte, cf. désormais Fabienne Jourdan, Poèmes attribués à Orphée, Paris : Les Belles Lettres, 2010). Le cœur du premier volume est donc constitué des fragments assignés à une œuvre connue sous le titre de Hieroi Logoi (« discours sacrés ») en 24 rhapsodies, la forme sous laquelle les néoplatoniciens tardifs (principalement Proclus et Damascius) ont connu la théogonie orphique. Bernabé préfère suivre la datation de cette théogonie au $\mathrm{I}^{\mathrm{er}}$ siècle avant J.-C., comme proposé par Martin L. West, plutôt que celle avancée par Luc Brisson à la fin du $\mathrm{I}^{\mathrm{er}}$ siècle, voire au début du II siècle de l'ère chrétienne, jugée plus vraisemblable par certains spécialistes, à l'instar de Radcliffe Edmunds III. Bernabé a renoncé à faire de la théogonie citée dans les Homélies pseudo-clémentines et celle, sensiblement différente, que l'on trouve dans les Reconnaissances du même pseudo-Clément une théogonie à part entière. Suivant Burkert et West, Bernabé y voit plutôt une forme allégorisée et quelque peu déformée de la théogonie des Rhapsodies. J'ai moi-même abordé cette question souvent négligée dans un article des Études classiques 76 (2008, publ. 2009), p. 83-94, « La cosmo-théogonie orphique du roman pseudo-clémentin: Note sur ses sources et son utilisation dans les Homélies et les Reconnaissances ", auquel Bernabé a répondu dans une étude publiée la même année dans Adamantius 14, p.79-99, «La teogonía órfica citada en las Pseudoclementina " (cf. aussi, plus récemment, Fabienne Jourdan, Orphée et les Chrétiens. La réception du mythe d'Orphée dans la littérature chrétienne grecque des cinq premiers siècles, 
Tome II : Pourquoi Orphée? (Anagôgê), Paris : Les Belles Lettres, 2011, p. 34-64). Signalons au passage que Bernabé a donné une utile traduction espagnole de toutes ces théogonies : Hieros logos. Poesía órfica sobre los dioses, el alma y el más allá, Madrid: Akal, 2003. Viennent ensuite (frg. 379-402) des poèmes orphiques consacrés à Déméter et Perséphone, parmi lesquels les fragments tirés du papyrus de Berlin $n^{\circ} 44$. Sont ensuite rassemblés des textes attribués également aux Pythagoriciens, dans lesquels le monde est comparé à un filet, à une tunique, à une sphère, à un cratère ou à une lyre. Ces textes précèdent des morceaux de poètes et de philosophes (frg. 421-469) consacrés à la nature de l'âme et au destin et que l'on peut raisonnablement rattacher à des traditions orphiques, à l'instar des plaquettes en os découvertes à Olbia, sur les bords de la mer Noire. C'est sur ces documents fascinants que s'achève le premier tome de cet opus magnum.

Le deuxième volume rassemble tout ce qui se rapporte aux rites orphiques (frg. 470-679) - parmi lesquels les lamelles d'or (frg. 474-496, dont Bernabé avait déjà livré, avec Ana Isabel Jiménez San Cristóbal et Ricardo Olmos, une édition critique avec traduction espagnole : Instrucciones para el más allá : las laminillas órficas de oro, Madrid, 2001, augmentée et traduite en anglais en 2008 [Instructions for the Netherworld. The Orphic Gold Tablets, Religions in the Graeco-Roman World 162, Leyde : Brill]) -, aux hymnes et aux épigrammes (frg. 680-705), aux catabases (frg. 707-717), aux matériaux à caractère astrologique (frg. 718-803), divinatoire (frg. 804-811) et magique (frg. 812-834), ainsi qu'aux témoignages sur Orphée (frg. 864-1095), ses disciples supposés (d'Eumolpe et Musée à Empédocle et Onomacrite) et les auteurs qui sont censés avoir écrit, utilisé ou commenté la poésie orphique (frg. 1096-1151).

Le troisième tome, paru en 2007, réunit des fragments et témoignages relatifs à des poètes plus ou moins directement liés à Orphée, tels Musée, Linus et Épiménide. Le volume offre en outre, en appendice, une édition critique du papyrus de Derveni, divers index (des mots, des sources, des auteurs et des œuvres), une table de concordance permettant de passer aisément de l'édition de Kern à celle de Bernabé, ainsi qu'une liste d'errata et d'addenda. La partie la plus importante de ce volume est donc constituée par le texte intégral du papyrus de Derveni, l'une des découvertes papyrologiques majeures de ces dernières décennies pour l'histoire des religions et la philosophie antique et pour laquelle nous disposons maintenant d'au moins deux éditions critiques dignes de ce nom, celle que Bernabé nous livre ici et celle de Theokritos Kouremenos, George M. Parássoglou, Kyriakos Tsantsanoglou, The Derveni Papyrus (Studi e testi per il "Corpus dei papiri filosofici greci e latini", vol. 13), Florence : Leo S. Olschki, 2006 (nombreux comptes rendus parus à ce jour), même si tout n'y est pas parfait et que des affinements ultérieurs devraient être apportés dans l'édition annoncée par Dirk Obbink, qui compte s'appuyer sur la technique de la photographie multispectrale pour mettre en lumière des points restés obscurs jusqu'ici. Signalons enfin qu'Alberto Bernabé est revenu sur les problèmes liés à l'édition du papyrus de Derveni lors d'un colloque de papyrologie qui s'est tenu aux Etats-Unis en 2007: "The Derveni Papyrus: Problems of Edition, Problems of Interpretation ", dans Proceedings of the Twenty-Fifth International Congress of Papyrology, Ann Arbor 2007 (American Studies in Papyrology, Ann Arbor 2010, p. 77-84).

En conclusion, cette édition des Orphica fragmenta et testimonia a les défauts de ses qualités. Bernabé visant à l'exhaustivité sur tous les plans - aussi bien dans les citations de textes, les apparats critiques que dans la bibliographie -, cela l'a conduit à multiplier les apparats, à les surcharger parfois de manière telle qu'ils s'étendent sur plusieurs 
pages, rendant la lecture malcommode et difficile. Ce même souci d'exhaustivité l'a aussi amené à reproduire les citations d'un même texte sous toutes les rubriques auxquelles il peut se rapporter. Ce dernier point a souvent été relevé par les critiques, mais l'éditeur ne peut vraiment en être tenu pour responsable, car cela tient à la nature même du matériel traité. La connaissance que Bernabé a de la littérature publiée sur ce sujet est si vaste qu'on en voit la trace presque à chaque page de cette édition, où il n'est pas de textes, de citations ou de variantes philologiques qui ne trouvent de renvois à une étude spécialisée, écrite dans l'une ou l'autre des langues modernes européennes. Cela donne à ce gigantesque travail une valeur inestimable, qui justifie à lui seul qu'on s'y réfère dès lors que l'on s'intéresse à Orphée et à l'orphisme. Aussi, malgré les petites imperfections qui jalonnent ces trois volumes, nul doute que cette édition fera date.

7 Pour saluer la parution de cette œuvre monumentale, plusieurs élèves et amis d'Alberto Bernabé (Miguel Herrero de Jáuregui, Ana Isabel Jiménez San Cristóbal, Eugenio R. Luján Martínez et d'autres) ont contribué à un volumineux collectif intitulé Tracing Orpheus: Studies in Orphic Fragments, Berlin: Walter De Gruyter, décembre 2011, dont nous recommandons également la lecture. Une année plus tôt, Bernabé avait fait paraître Orfeo y el orfismo: nuevas perspectivas, Alicante: Biblioteca Virtual Miguel de Cervantes, en collaboration avec F. Casadesús Bordoy, M. A. Santamaría Álvarez et, en 2009, Orfeo y la tradición órfica. Un reencuentro, Madrid : Akal, en deux volumes coédités avec le même F. Casadesús.

\section{AUTEURS}

\section{JEAN-MICHEL ROESSLI}

Concordia University, Montréal, Canada 